\title{
Review Article: Inventory of platforms towards the design of a statically balanced six degrees of freedom compliant precision stage
}

\author{
A. G. Dunning, N. Tolou, and J. L. Herder \\ Faculty of Mechanical, Maritime and Materials Engineering, Department of Biomechanical Engineering, \\ Delft University of Technology, Delft, The Netherlands
}

Received: 1 March 2011 - Revised: 9 June 2011 - Accepted: 26 July 2011 - Published: 4 August 2011

\begin{abstract}
For many applications in precision engineering, a six degrees of freedom (DoF) compliant stage (CS) with zero stiffness is desirable, to deal with problems like backlash, friction, lubrication, and at the same time, reduce the actuation force. To this end, the compliant stage (also known as compliant mechanism) can be statically balanced with a stiffness compensation mechanism, to compensate the energy stored in the compliant parts, resulting in a statically balanced compliant stage (SBCS). Statically balanced compliant stages can be a breakthrough in precision engineering. This paper presents an inventory of platforms suitable for the design of a $6 \mathrm{DoF}$ compliant stage for precision engineering. A literature review on 3-6 DoF compliant stages, static balancing strategies and statically balanced compliant mechanisms (SBCMs) has been performed. A classification from the inventory has been made and followed up by discussion. An obviously superior architecture for a 6 DoF compliant stage was not found. All the $6 \mathrm{DoF}$ stages are either non-statically balanced compliant structures or statically balanced non-compliant structures. The statically balanced non-compliant structures can be transformed into compliant structures using lumped compliance, while all SBCMs had distributed compliance. A 6 DoF SBCS is a great scope for improvements in precision engineering stages.
\end{abstract}

\section{Introduction}

Many applications in precision engineering, including lithography, electron beam microscopy, micro assembly, aerospace, medical applications, require ultra precision positioning to manipulate an object in a vacuum or wet environment. For instance, in lithography the electrical circuits written on a wafer will have a resolution smaller than $20 \mathrm{~nm}$ (Willson and Roman, 2008). In the medical field, precise surgical tools with good force feedback are required to avoid tissue damage during operation (Sjoerdsma et al., 1997). All the named applications are situated inside a vacuum or wet environment. Therefore it is difficult to use conventional bearings, due to the need of lubrication. The backlash in conventional joints also has been an issue in high precision engineering. To overcome these problems, compliant mechanisms can be used.

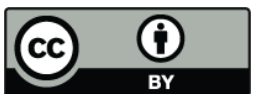

Correspondence to: J. L. Herder (j.1.herder@tudelft.nl)
A compliant mechanism is a mechanism that transfers force, motion or energy by using the elastic deformation of its flexible components rather than using rigid-body joints only. An advantage of compliant mechanisms is that it can easily be manufactured as a monolithic structure due to its hingeless nature of the design. This absence of movable joints reduces wear, friction and backlash in the mechanism and correspondingly increases precision, which is an important factor in the design of high-precision instrumentation. There is also no need for lubrication and the mechanism is insensitive to dust, which is an important advantage in instruments under vacuum (Howell, 2001).

However, the compliant mechanisms rely on the deflection of flexible members, which introduces positive stiffness and requires energy to deform. Therefore, the energy storage in the flexible members is distorting the input-output relationship and challenges the mechanical efficiency. When the deformation of the flexible members is large, non-linearities are introduced, which increases the complexity of the design (Herder and van den Berg, 2000; Morsch and Herder, 2010). 
In many of the mentioned fields, it is required to manipulate an object in six degrees of freedom (DoF). In particular, in lithography and electron beam microscopy, the actuation of the 6 DoF positioning stage produces too much heat, mainly caused by the stiffness of the stage, which can affect the precision of the application (Nieuwenhuis, 2010). In medical instruments, the force feedback is not optimal, due to the stiffness and friction introduced in compliant and contact members (Sjoerdsma et al., 1997).

To overcome these problems a stiffness compensation mechanism can be added to the compliant mechanism, resulting in a statically balanced compliant mechanism (SBCM) with nearly zero stiffness. A statically balanced mechanism (SBM) is a mechanism on which the forces of one or more potential energy storage elements are acting, such that the mechanism is in static equilibrium and therefore has zero stiffness. The total potential energy should be constant in every position of the mechanism (Herder, 2001). To create static balancing a positive stiffness of the mechanism should be balanced with a negative stiffness compensation device. Therefore, it can be very advantageous to integrate a $6 \mathrm{DoF}$ SBCM into an available application and replace the conventional positioning system.

The purpose of this literature survey consists of (1) to provide an overview of the state of the art of $6 \mathrm{DoF}$ compliant stages. Interesting stages with less degrees of freedom, where translations are combined with rotations have also been investigated. A classification is made to compare the available stages to investigate whether there is a superior design for $6 \mathrm{DoF}$ compliant stages. Thereafter, (2) an inventory on balancing strategies for compliant mechanisms is made. Finally, (3) possibilities to combine a $6 \mathrm{DoF}$ compliant stage with static balancing will be investigated.

In Sect. 2, the method, including search method, search criteria, and the method to classify the results, is explained. The results of the literature survey are briefly described in Sect. 3. In Sect. 3.1 the results of the 6 DoF compliant stages are presented. It presents the type and classification of flexures, serial and planar positioning structures. Section 3.2 describes the balancing strategies with existing SBCMs and structures combining 6 DoF with static balancing. Section 4 interprets and discusses the results of each goal. Conclusions are presented in Sect. 5 .

\section{Method}

\subsection{Search method}

The literature survey is separated into two parts. In the first part a literature search is conducted for $6 \mathrm{DoF}$ compliant precision stages. This part also considers stages with fewer DoFs that may be converted into 6 DoF. These are stages with 3,4 or 5 degrees of freedom, where translational degrees of freedom were combined with rotational degrees of freedom.
The second part is to examine the static balancing strategies for compliant mechanisms and make a classification.

By analyzing the topics a search plan was made. The key subjects and constraints were determined, particularly in the field of precision engineering. Only stages with a motion smaller than $1 \mathrm{~mm}$ were searched for. Subsequently, key subjects were transformed into search terms, comprising synonyms and related terms. These search terms were used in the set of keywords in the search engines.

In total five different sets of keywords have been used, concerning keywords defining (1) compliant mechanisms, (2) the field of precision engineering, (3) $6 \mathrm{DoF}$ stages, (4) static balancing and (5) zero stiffness.

In order to optimize the search, all sets of keywords were combined and narrowed. Also the references of the articles were checked for useful articles in the same subject. The results were first filtered by inspecting the article titles. Subsequently, the reduced results were filtered by reading the abstracts and looking to the images in the article. From the abstract or the images the working principle needed to be clear. Otherwise the papers were discarded.

The literature search was conducted using two search engines (Scopus; Espacenet). SCOPUS was used for journal articles and conference proceedings, while Espacenet was used to search for patents. All five sets of keywords were used in SCOPUS. Espacenet is the search engine of the European Patent Office and searches patents from all over the world. This engine is able to search patents with a set of keywords, instead of a classification system. Only patents of $6 \mathrm{DoF}$ compliant stages and SBCMs were of interest for this literature survey, only specific combinations of sets of keywords were used. An overview of the sets of keywords can be found in Table 1.

\subsection{Classification}

A classification was made to compare the results of the compliant mechanisms within the field of $6 \mathrm{DoF}$ stages and precision engineering. The following strategy and criteria have been used for classification.

The first and second level of classification, indicated the architecture of the mechanism. In the first level, a distinction was made between planar and spatial geometry of the structure. In a planar structure, in contrast to spatial structures, flexible elements to perform a $6 \mathrm{DoF}$ motion are in the same plane, so for some motion out-of-plane motion is required. The second level described the configuration of the kinematic chain mechanism. This can be a parallel or a serial configuration (Lobontiu, 2003). In a parallel configuration, also called a closed-loop configuration, the fixed base is connected to the movable end-effector through multiple kinematic chains. A good example of a parallel mechanism is the Stewart platform (Stewart, 1965). Serial mechanisms use an open loop serial chain of links to connect the base with the end-effector. A robot arm is a good example of a serial mechanism. 
Table 1. Overview of the sets of keywords used in SCOPUS (1-5) and Espacenet $(1,3,4,5)$.

\begin{tabular}{ll}
\hline Sets & Keywords \\
\hline (1) Compliant mechanisms & - Compliant, flexible, flexure, monolithic \\
& - Mechanism, structure, design \\
\hline (2) Precision engineering & - Precision, micro, nano, sensible \\
& - Stage \\
\hline (3) 6 DoF stage & - Six degrees of freedom, six axis \\
& - Stage \\
\hline (4) Static balancing & - Static balancing, neutral equilibrium \\
\hline (5) Zero stiffness & - Zero/neutral/eliminate/remove/cancel stiffness \\
& - Constant potential energy, pre-stressed \\
& - Neutral stability \\
& - Gravity compensation \\
\hline
\end{tabular}

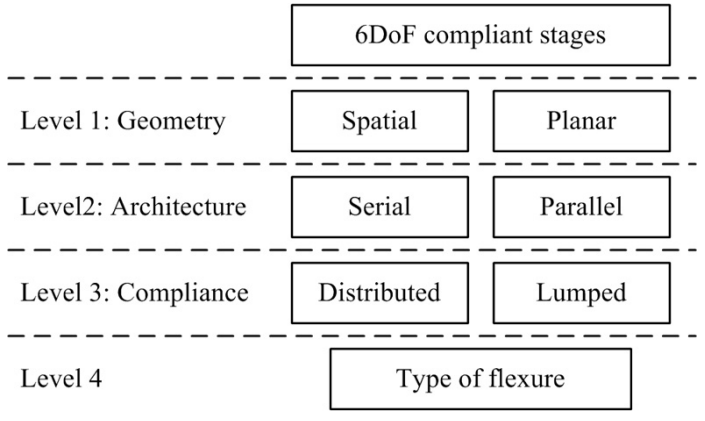

Figure 1. Schematic representation of the classification levels to compare the $6 \mathrm{DoF}$ compliant stages.

The third level of classification described the types of stress distribution in the mechanism, which are lumped compliance and distributed compliance (Ananthasuresh and Kota, 1995).

In the fourth level the type of flexures used in the mechanism was distinguished.

In Fig. 1, a schematic representation of the classification is provided. Quantitative data found, involving size (S), working range (WR), will be noted.

To compare the stages, the ratios between translations, rotations and the size of the stages were investigated.

The SBCMs were classified according to the balancing principle, using (1) counterweights or (2) elastic elements, to compensate gravity forces or strain energy inside the mechanism (Herder, 2001). The mechanisms in these categories can be classified further according to the type of compensation mechanism. If reported in the article, the remaining stiffness after balancing, the statically balanced stroke and the size of the balancing mechanism is mentioned.

\section{Results}

\subsection{State of the art in 6 DoF compliant stages}

In the field of precision engineering the demand for $6 \mathrm{DoF}$ stages is high. These stages have to be very accurate, with a resolution in the order of nanometers (Willson and Roman, 2008). In literature, precision compliant stages, which combine translations and rotations, with 3,5 and $6 \mathrm{DoF}$ were found. All the $6 \mathrm{DoF}$ stages had three translational $(\mathrm{x}, \mathrm{y}, \mathrm{z})$ and three rotational $(\theta \mathrm{x}, \theta \mathrm{y}, \theta \mathrm{z})$ degrees of freedom. One 5 DoF stage (Wang et al., 2005) was found, which had no degree of freedom in rotation around the z-axis, and the $3 \mathrm{DoF}$ stages had all two translational $(\mathrm{x}, \mathrm{y})$ and one rotational $(\theta \mathrm{z})$ degrees of freedom. All the designs found in literature were fully compliant. In other words, no conventional joints were used for transferring motion. Besides, all the designs were highly symmetric, otherwise it is mentioned.

An overview of all the available results, including flexure type, size (S) and working range (WR) is shown in Table 2.

\subsubsection{Type of flexures}

Different flexures were found in the compliant mechanisms. Depending on the characteristics of the flexure it can have single or multiple deflection axes, which can be translational or rotational. Two rotational deflection axes in a joint create a universal joint and a combination of three rotational joints creates a spherical joint.

The flexible components could be classified in two groups, with flexures having (1) lumped compliance and (2) distributed compliance. With lumped compliance the flexion concentrates around a distinct number of flexures, causing high stress concentrations in the mechanism. These flexible elements have also low static and fatigue strength, usually undergoes small displacements, and manufacturing 
Table 2. Overview of the results of the compliant stages, mentioned flexure type (mentioned with •), size and working range.

Data not available identified with -.

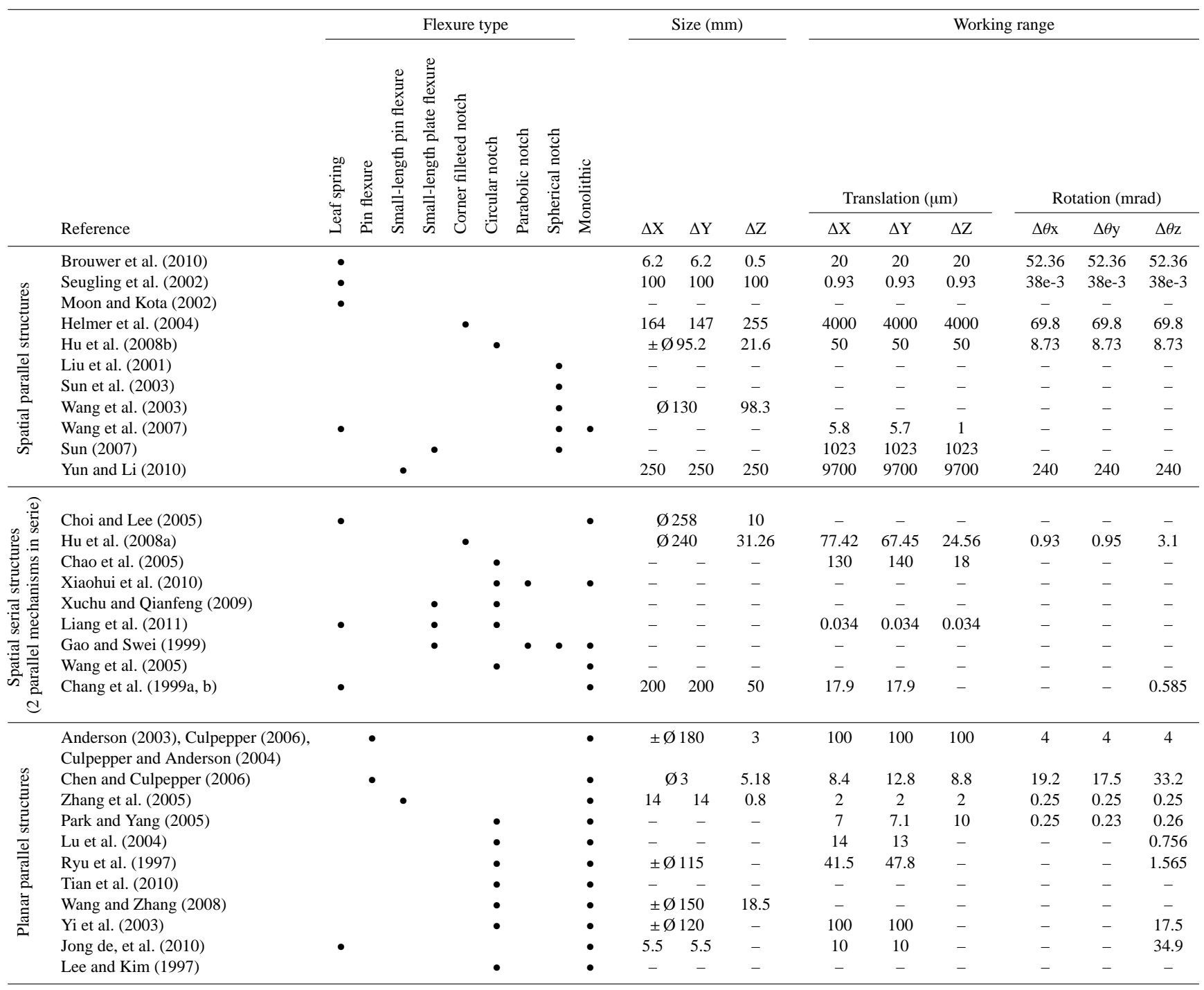

these elements can give difficulties, due to very thin sections (Ananthasuresh and Kota, 1995; Gallego and Herder, 2009). In this group, notch-type flexures and small-length plate and pin flexures could be found. The notch profile could be a (1) rectangular corner-filleted, (2) circular, (3) parabolic, or (4) spherical section (Fig. 2). The small-length plate flexure could bend in one degree of freedom and the pin flexure could bend in all three rotational degrees of freedom (Gallego and Herder, 2009).

For distributed compliant flexures, the flexibility is distributed equally over the entire flexible element. The flexible element has a constant cross-section, which prevent stress concentration around a point. Distributed compliance offers better performance and reliability compared to lumped compliance (Ananthasuresh and Kota, 1995). The pin flexure could bend in all three rotational degrees of freedom and
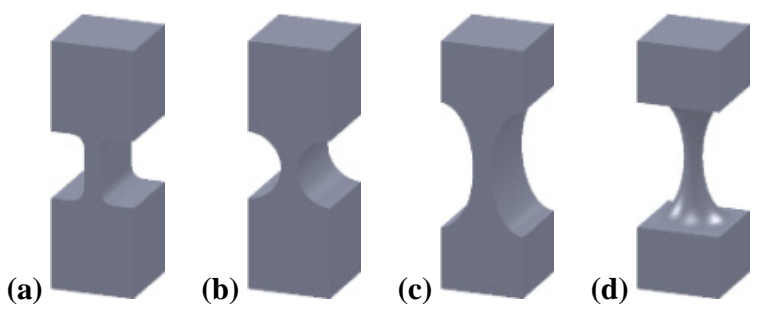

Figure 2. Notch-type flexures with lumped compliance. The notch profile is (a) rectangular corner-filleted, (b) circular, (c) parabolic, or (d) spherical. 
(a)

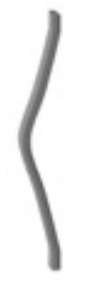

(b)

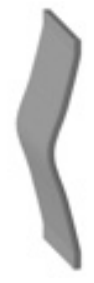

(c)

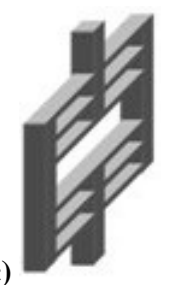

(d)

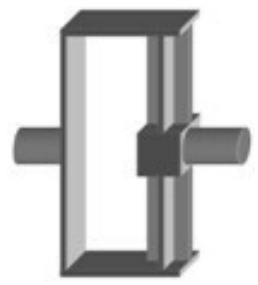

(e)

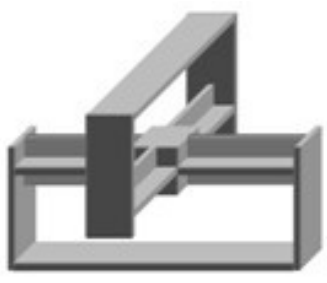

(f)

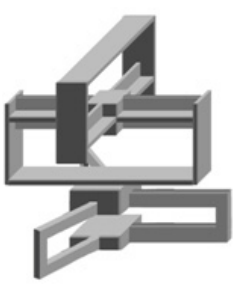

Figure 3. Flexures with distributed compliance. The flexures could be a (a) pin, (b) chevron, (c) translational, (d) rotational, (e) universal, or (f) spherical flexure. Reproduced from Gallego and Herder (2009).

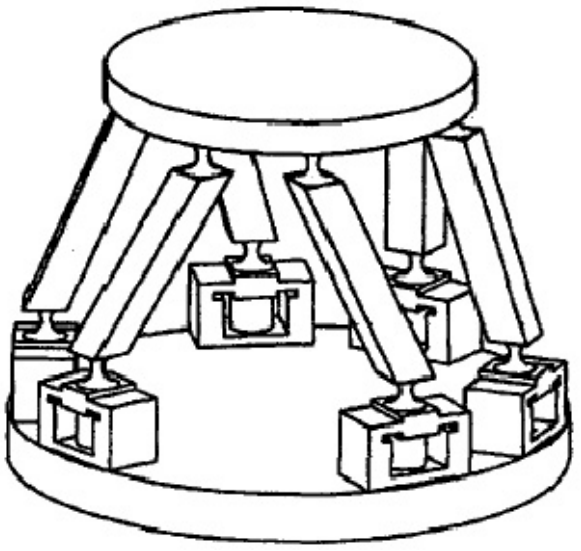

Figure 4. Typically example of a spatial parallel compliant stage (Liu et al., 2001). The platform is supported by legs, with compliant joints at both ends.

a chevron flexure, also called a leaf spring, could bend in one direction and take up torsion. Almost all of these flexures were built up from combining several chevron flexures in such a way that joints with different degrees of freedom are possible (Fig. 3) (Gallego and Herder, 2009).

\subsubsection{Spatial compliant stages}

The results for spatial compliant stages were separated into a group with a parallel and a serial kinematic chain. First the parallel designs will be described (Fig. 4).

In Brouwer et al. (2010) in-plane leaf springs form prismatic joints and three slanted leaf springs for out-of-plane motion form three universal joints. The flexures, arranged by $120^{\circ}$, create a monolithic spatial parallel platform stage. The same kind of flexures are used in Seugling et al. (2002) and Moon and Kota (2002). In the latter article, the leaf springs were combined such that they form a prismatic, rotational and spherical joint, respectively.

A large non-symmetric stage with corner-filleted notches was developed in Helmer et al. (2004).

Circular notch-type flexures are used in $\mathrm{Hu}$ et al. (2008b). Here six slanted trapeziform displacement amplifiers form a spatial stage. Each trapeziform amplifier can be modeled as two prismatic joints.

Spherical notches were found in mechanisms based on the Stewart platform. In Liu et al. (2001), Sun et al. (2003), and Wang et al. (2003) the platform is supported by 6 legs, that is the compliant equivalent of a 6-spherical-prismaticspherical manipulator. In Wang et al. (2007) the platform is supported by 3 legs. Each leg is the compliant equivalent of a rotational-spherical manipulator. The legs are placed on small compliant mechanisms, which enables translational motion in $2 \mathrm{DoF}$ with leaf springs and are placed $120^{\circ}$ of each other.

Sun (2007) used a non-symmetric stage with spherical notch-type flexures in series with small-length plate flexures (prismatic joints) to create the desired degrees of freedom.

In Yun and Li (2010) small-length pin flexures on both sides of an actuator are used to move a platform. In total eight non-symmetrically placed actuators are used, which makes the stage the compliant equivalent of a 8-prismatic-sphericalspherical/spherical-prismatic-spherical manipulator.

All stages with a serial kinematic chain were constructed as two parallel mechanisms in series, a so-called serialparallel mechanism (Fig. 5). All stages consist of a parallel monolithic mechanism, which could perform the motion in $\mathrm{x}, \mathrm{y}$ and $\theta \mathrm{z}$ direction (further mentioned as in-plane motion), and a parallel mechanism performing motion in $\mathrm{z}, \theta \mathrm{x}$, $\theta \mathrm{y}$ direction (further mentioned as out-of-plane motion). The flexures are all arranged $120^{\circ}$ of each other.

Choi and Lee (2005) designed a stage where the motion is enabled by leaf springs. The $\mathrm{x}, \mathrm{y}$ and $\theta \mathrm{z}$ motions are transferred by six L-shaped leaf springs and the $\mathrm{z}, \theta \mathrm{x}, \theta \mathrm{y}$ motions are transferred by wide leaf springs.

In $\mathrm{Hu}$ et al. (2008a) the flexures are cornered-filleted notches. The in-plane mechanism is the compliant equivalent of a traditional 3-revolute-revolute-revolute manipulator. The out-of-plane mechanism is an equivalent of a traditional 3-universal-prismatic-universal manipulator.

Chao et al. (2005) used a 3-revolute-revolute-revolute compliant mechanism with circular notches for the in-plane motion. For the out-of-plane motion a 3-revolute-prismaticspherical compliant mechanism with circular notches is used to form 3 legs, supporting the moving platform. The stage 


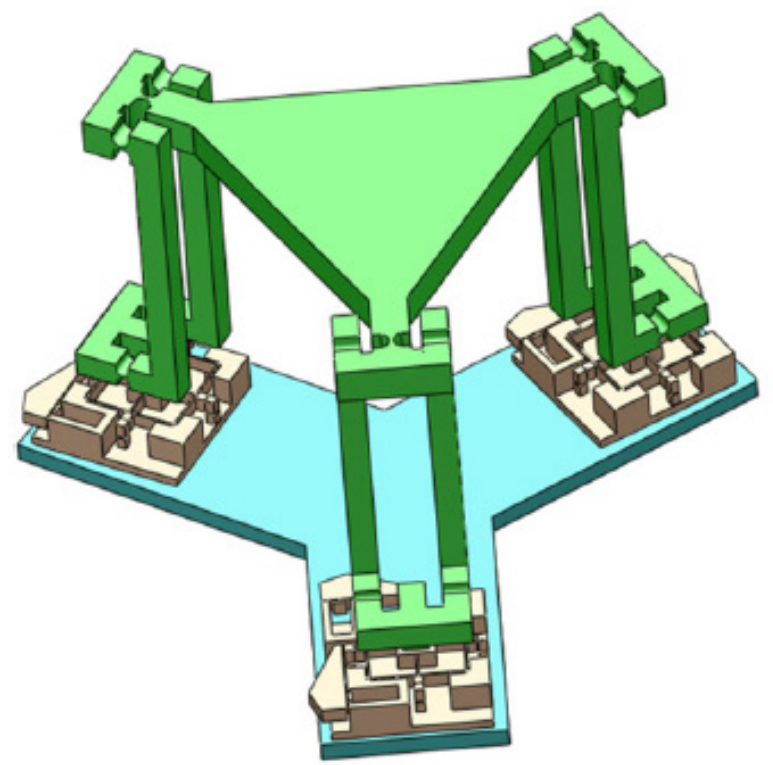

Figure 5. Typically example of a spatial serial compliant stage (Liang et al., 2011). Three legs forms a parallel compliant mechanism performing motion in $\mathrm{z}, \theta \mathrm{x}, \theta \mathrm{y}, \theta \mathrm{z}$. The legs are supported by parallel 2 DoF compliant mechanisms. Both parallel mechanisms in serie forms the spatial serial compliant stage.

from Xiaohui et al. (2010) has the same compliant equivalent structure as Chao et al. (2005) for in-plane motion. The outof-plane motion is performed by 3 parabolic notch-type flexures. In Xuchu and Qianfeng (2009) a 3-revolute-revolute compliant mechanism with circular notches is used for inplane motion. Small-length plate flexures are used for the out-of-plane motion.

Liang et al. (2011) used 3 legs, each consisting of two universal joints, supporting a platform for out-of-plane motion with $4 \operatorname{DoF}(\mathrm{z}, \theta \mathrm{x}, \theta \mathrm{y}, \theta \mathrm{z})$. These universal joints were manufactured with circular notch-type flexures. The in-plane motion $(\mathrm{x}, \mathrm{y})$ is provided by a spatial mechanism consisting of small-length plate flexures and leaf springs.

In Gao and Swei (1999) the compliant equivalent of a 3revolute-prismatic-revolute manipulator is used for in-plane motion and a 3-revolute-prismatic-spherical manipulator for the out-of-plane motion. Three legs, with a parabolic and a spherical notch-type flexure, support the platform. The inplane motion is provided by small-length plate flexures.

Wang et al. (2005) developed a 5 DoF compliant stage made with circular notch-type flexures, having a monolithic mechanism to provide translation along the $\mathrm{x}$-axis and $\mathrm{y}$-axis and a 4-revolute-revolute compliant mechanism to provide translation along the z-axis and rotations in all directions. The flexures in this stage are not arranged $120^{\circ}$ of each other.

Chang et al. (1999a, b) designed a 3 DoF stage with leaf springs and small-length plate flexures, consisting of a $2 \mathrm{DoF}$ $(\mathrm{x}, \mathrm{y})$ stage and a $1 \mathrm{DoF}(\theta \mathrm{z})$ stage on top of it, which makes it also a serial-parallel structure.

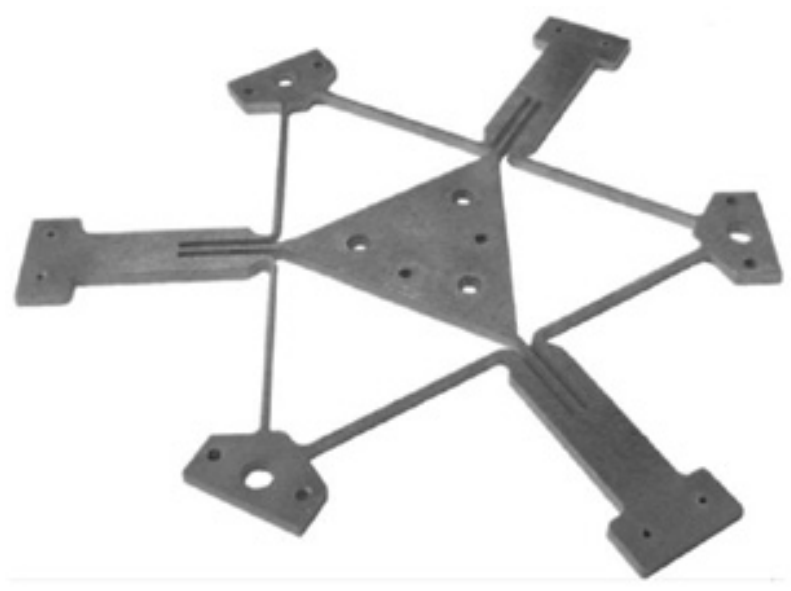

Figure 6. Typically example of a planar compliant stage (Anderson, 2003; Culpepper, 2006; Culpepper and Anderson, 2004). The flexures to perfom motion are in the same plane.

\subsubsection{Planar compliant stages}

Only a few stages have a planar structure (Fig. 6). The main advantage of planar structures is that the whole mechanism can be manufactured monolithic and have a high stiffness, but usually a small workspace, compared to serial mechanisms. All the planar designs found in the articles were monolithic, and had a parallel kinematic chain. The differences in each design were the used flexure type.

In Anderson (2003), Culpepper (2006), and Culpepper and Anderson (2004) a nano-manipulator, called the HexFlex, which use 3 long pin flexures, placed $120^{\circ}$ to each other, to enable $6 \mathrm{DoF}$ is presented. Each flexure enables in-plane and out-of-plane motion. In Chen and Culpepper (2006) and Culpepper and Golda (2007) two different types of mircoscaled versions of the HexFlex are made. In Zhang et al. (2005) the 6 DoF motion is enabled by four parallelograms. With small-length pin flexures the parallelograms can move in-plane and out-of-plane. In Park and Yang (2005) a set of circular notches arranged by $120^{\circ}$ creates in-plane motion, and inclined circular notches placed $45^{\circ}$ with respect to the plane enables out-of-plane motion.

Planar monolithic $3 \mathrm{DoF}$ stages were found in $\mathrm{Lu}$ et al. (2004), Ryu et al. (1997), Tian et al. (2010), Wang and Zhang (2008), and Yi et al. (2003). The circular notch flexure groups are arranged $120^{\circ}$ of each other. All the designs are modeled with a 3-revolute-revolute-revolute manipulator. Almost the same structure was found in a MEMSbased manipulator, produced by Jong de, et al. (2010), but the flexures are leaf springs and the compliant equivalent of a 3-prismatic-revolute-revolute manipulator is used. Lee and Kim (1997) designed an ultra-precision micro stage, with circular notch flexures, to correct the errors of a global stage. 
Table 3. Overview of the results of the statically balanced compliant mechanisms (SBCM) and 6 DoF statically balanced mechanisms (SBM). The balancing mechanism is either with counterweights (C) or elastic elements, using springs (S), zero-free-length springs (ZFLS) or compliant flexures $(\mathrm{CF})$, which are categorized into the use of buckling plates (BP), preloaded plates (PP), to balance strain energy (E) or gravity forces (G). Data not available identified with -.

\begin{tabular}{|c|c|c|c|c|c|c|c|c|c|c|c|c|c|}
\hline & \multirow[b]{2}{*}{ Reference } & \multicolumn{4}{|c|}{ Flexure type } & \multirow{2}{*}{\multicolumn{2}{|c|}{ 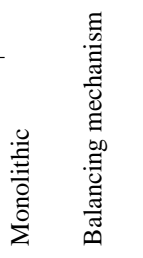 }} & \multirow[b]{2}{*}{$\begin{array}{l}\stackrel{00}{\Xi} \\
\stackrel{\Xi}{\Xi} \\
\frac{0}{D} \\
D \\
D\end{array}$} & \multirow[b]{2}{*}{ 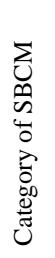 } & \multirow[b]{2}{*}{ 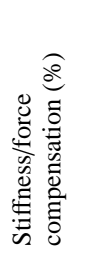 } & \multirow{2}{*}{ 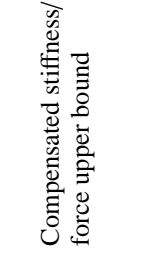 } & \multirow[b]{2}{*}{ 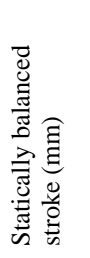 } & \multirow{2}{*}{ 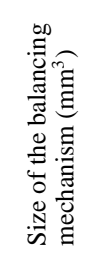 } \\
\hline & & 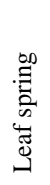 & 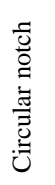 & 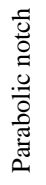 & 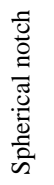 & & & & & & & & \\
\hline \multirow{11}{*}{$\sum_{\substack{\infty \\
\infty}}$} & Eijk van, and Dijksman (1979) & $\bullet$ & & & & & $\mathrm{BP}$ & $\mathrm{E}$ & - & $100 \%$ & - & - & - \\
\hline & Herder and van den Berg (2000) & $\bullet$ & & & & & $\mathrm{S}$ & $\mathrm{E}$ & 1 & $99.9 \%$ & $12.9 \mathrm{~N}$ & 1 & $\pm 49 \times 10^{3}$ \\
\hline & Stapel and Herder (2004) & $\bullet$ & & & & $\bullet$ & PP & $\mathrm{E}$ & 3 & $100 \%$ & $\pm 50 \mathrm{~N} \mathrm{~mm}^{-1}$ & 0.3 & \pm 4280 \\
\hline & Tolou and Herder (2009) & $\bullet$ & & & & & PP & $\mathrm{E}$ & 3 & $100 \%$ & $19 \mathrm{~N}$ & 4.17 & \pm 720 \\
\hline & Lange de, et al. (2008) & $\bullet$ & & & & $\bullet$ & $\mathrm{BP}$ & $\mathrm{E}$ & 3 & $90 \%$ & $300 \mathrm{~N}$ & 0.65 & \pm 980 \\
\hline & Powell and Frecker (2005) & $\bullet$ & & & & & $\mathrm{S}$ & $\mathrm{E}$ & 1 & $100 \%$ & - & - & - \\
\hline & Hoetmer et al. (2009) & $\bullet$ & & & & $\bullet$ & BP & $\mathrm{E}$ & 3 & $120 \% *$ & $1 \mathrm{~N} \mathrm{~mm}^{-1}$ & 1.7 & \pm 1850 \\
\hline & Morsch and Herder (2010) & $\bullet$ & & & & & $\mathrm{PP}$ & $\mathrm{E}$ & 3 & $70 \%$ & $6.5 \mathrm{~N}^{* *}$ & $23.6^{* * *}$ & $\pm 4 \times 10^{5}$ \\
\hline & Trease and Dede (2004) & $\bullet$ & & & & & $\mathrm{CF}$ & $\mathrm{G}$ & 3 & $100 \%$ & $\pm 5 \mathrm{~N}$ & - & - \\
\hline & Tolou and Herder (2010) (case I) & $\bullet$ & & & & $\bullet$ & BP & $\mathrm{E}$ & 3 & $99 \%$ & $60 \mathrm{mN}$ & 0.05 & 9.6 \\
\hline & Tolou and Herder (2010) (case II) & $\bullet$ & & & & $\bullet$ & $\mathrm{BP}$ & $\mathrm{E}$ & 3 & $86 \%$ & $40 \mathrm{mN}$ & 0.06 & 1.6 \\
\hline \multirow{5}{*}{ 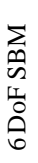 } & Streit (1991) & & & & $\bullet$ & & ZFLS & G & & & & & \\
\hline & Ebert-Uphoff and Johnson (2002), Ebert-Uphoff et al. (2000) & & & & $\bullet$ & & S & $\mathrm{G}$ & & & & & \\
\hline & Gosselin and Wang (2000) & & & & & & $\mathrm{C}, \mathrm{S}$ & G & & & & & \\
\hline & Leblond and Gosselin (1998) & & & & & & $\mathrm{S}$ & G & & & & & \\
\hline & Shekarforoush et al. (2010) & & & & $\bullet$ & & ZFLS & $\mathrm{G}$ & & & & & \\
\hline
\end{tabular}

* This mechanism is overcompensated.

** Compensated force is calculated from given compensated moment

*** Stroke is calculated from stroke given in radian.

\subsection{Static balancing strategies for compliant mechanisms}

Static balancing can be classified according to the balancing principle (Herder, 2001). These balancing principles are: (1) the addition of counterweights and (2) the use of elastic elements, to compensate gravity forces or strain energy inside the mechanism.

With the use of counterweights, the system is in equilibrium in any position. This method adds extra mass and inertia to the system, relative to springs or other elastic elements. The total potential energy of all gravity and elastic elements must be constant for perfect static balance.

There are several categories of SBCMs. These include (1) a compliant part balanced with a non-compliant compensation mechanism, (2) a compliant part with a compliant balancing mechanism, where the energy is stored in a separate spring, (3) the compensation energy is stored in a compliant part of the mechanism, rather than in a separate spring, and (4) adaptive balancing, taking into account that compliant mechanisms behave different under loaded and unloaded situations (Herder and van den Berg, 2000).

In Table 3 an overview of the results can be found.

\subsubsection{Statically balanced compliant mechanisms}

In literature, examples of SBCMs using elastic elements are very rare. In Eijk van, and Dijksman (1979) a mechanism with a constant negative stiffness, using a buckled plate spring, has been studied. Herder and van den Berg (2000) compensate the undesired stiffness in a laparoscopic grasper with a rolling-link mechanism and conventional helical springs (category 1). The reduced stiffness is in the order of $0.1 \%$ of the stiffness of the gripper. In Stapel and Herder (2004) a fully compliant compensation device, based on a slider-rocker mechanism, for the laparoscopic grasper is developed (category 3 ). The total potential energy in the system is almost constant. In Tolou and Herder (2009), the gripper of Herder and van den Berg (2000) is balanced with a partially compliant mechanism, consisting of pairs of pre-stressed pinned-pinned initially curved beams, arranged perpendicular to the link driving the grasper and placed inside the tip of the grasper (category 3 ). This resulted in force of almost $0 \mathrm{~N}$ to operate the grasper. Lange de, et al. (2008) used topology optimization to design a fully compliant grasper with a bistable balancing mechanism, with an actuation force reduction of $90 \%$, but due to calculated high stresses, a prototype is never fabricated (category 3). Powell and Frecker (2005) balanced a compliant forceps with a rigid link slider-crank 
mechanism with a non-linear spring, optimized with potential energy analysis with finite element analysis (category 1 ).

Hoetmer et al. (2009) used the Building Block Approach to balance a gripper. With the use of a new balanced building block, consisting of buckling plates, the stiffness was reduced from $1 \mathrm{~N} \mathrm{~mm}^{-1}$ to $-0.2 \mathrm{~N} \mathrm{~mm}^{-1}$ (category 3 ).

In Morsch and Herder (2010), the joint of a conventional balanced mechanism (Herder, 2001) is replaced by a crossaxis flexural pivot, and the zero-free-length springs by compliant leaf springs (category 3 ). This resulted in a fully compliant joint with a moment reduction of $70 \%$, measured from experiments.

Trease and Dede (2004) designed a partially compliant four bar mechanism with novel "open-cross" compliant joints to form a torsion-spring-based statically balanced gravity compensator (category 3 ). The potential energy of the system was balanced over $\pm 45^{\circ}$ from horizontal plane within a $3 \%$ error.

In Tolou and Herder (2010), two different statically balanced compliant micro mechanisms were designed (category 3) where the preloading force and stroke are either perpendicular or collinear. The first type compensated the force for $99 \%$ in the beginning of the travel path, due to external preloading force. But the collinear-type has been internally balanced without separated external preloading force, by using a bi-stable mechanism, compensating the force for $86 \%$ at the end of the stroke.

All the above-mentioned SBCMs had one degree of freedom and had distributed compliance. The design methods may well be used to implement in a $6 \mathrm{DoF}$ structure.

\subsubsection{DoF statically balanced mechanisms}

In literature $6 \mathrm{DoF}$ SBCMs is not readily available. An investigation of the possibilities to combine compliant mechanisms with static balancing some 6 DoF SBMs found in literature are discussed here. All the structures discussed here are spatial parallel platform mechanisms.

Streit (1991) presented the first 6 DoF SBM. He presented a parallel platform mechanism consisting of three legs, where each leg has three degrees of freedom. The legs are parallelograms connected to the platform with spherical joints, and balanced with zero-free-length springs. Static balancing is only achieved when the centre of mass of the platform is close to the plane of the spherical joints. In Ebert-Uphoff and Johnson (2002) and Ebert-Uphoff et al. (2000) this condition is removed by introducing pulling and pushing legs connected to the platform with spherical joints. The mechanism has three active pushing legs, which tilt the platform upwards, and one passive pulling leg, attached in slightly offcentre of the platform and pulling the platform down to a static balanced condition.

Gosselin and Wang (2000) used six legs with revolute actuators to balance a platform, using both the counterweights method and the spring method.
Leblond and Gosselin (1998) showed different ways to balance existing spatial parallel mechanisms, such as the Gough-Stewart platform, with additional elements.

Shekarforoush et al. (2010) balanced two types of $6 \mathrm{DoF}$ tensegrity systems, with passive zero-free-length springs and with an adjustable cable-spring combination. The connection between legs and the platform are all ball-socket joints, which could be represented as spherical joints.

In Table 3 the results are shown for balancing principle and which compliant flexure type could represent the joints in the mechanisms.

\section{Discussion}

In this part, the results are compared and discussed with each other based on criteria. Many articles did not mention size or working range, which makes it a challenge to compare all stages with each other. Besides, not every stage had the same structure to make a good comparison. Therefore, a comparison between all planar structures is made and finally the spatial stages are compared.

To make a good comparison, the ratios between translations, rotations and the size of the stages are compared. The ratios are normalized to the largest in the group, as shown in Fig. 7.

First, the ratios of translations (in $\mu \mathrm{m}$ ) in the XY-plane relative to the size (in $\mathrm{mm}$ ) of the XY-plane of planar structures $(\mathrm{WRx} \cdot \mathrm{y} / \mathrm{Sx} \cdot \mathrm{y})$ are compared. It is noteworthy, that in Chen and Culpepper (2006) the largest ratio is reached. Considering the ratios between rotations (in mrad) around the $\mathrm{z}$-axis and the size (in $\mathrm{mm}$ ) in the XY-plane (WR $\theta z / \mathrm{Sx} \cdot \mathrm{y})$, again the largest ratio has been reached in Chen and Culpepper (2006). Also in Jong de, et al. (2010) and Ryu et al. (1997) a relative large ratio is found, compared to the other stages. The results showed that there is no clear relation between flexure type and translation/size or rotation/size ratio in XY-plane. Both Chen and Culpepper (2006) and Chang et al. (1999a) used leaf springs, but had the largest and the smallest ratios, respectively. Also the notch-type flexures did not showed ratios in the same order.

For the spatial stages the ratios of working range of the translations (in $\mu \mathrm{m}$ ) relative to the size (in $\mathrm{mm}$ ) of the stage (WRx $\cdot y \cdot z / S x \cdot y \cdot z)$ shows that the stage from Seugling et al. (2002) has a very small working range with respect to the size. In Brouwer et al. (2010), Culpepper and Anderson (2004), and Chen and Culpepper (2006) the ratios are high, due to the almost planar structure of the stages, which are able to perform $6 \mathrm{DoF}$ motion. But the largest ratio is reached by a spatial structure (Yun and $\mathrm{Li}, 2010$ ). Comparing the ratios between rotations (in mrad) and size (in $\mathrm{mm})(\mathrm{WR} \theta \mathrm{x} \cdot \theta \mathrm{y} \cdot \theta \mathrm{z} / \mathrm{Sx} \cdot \mathrm{y} \cdot \mathrm{z})$ shows high ratios in Brouwer et al. (2010) and Chen and Culpepper (2006). This is also due to their planar structure. Remarkably, the ratio of the planar stage in Culpepper and Anderson (2004) is not as high as 


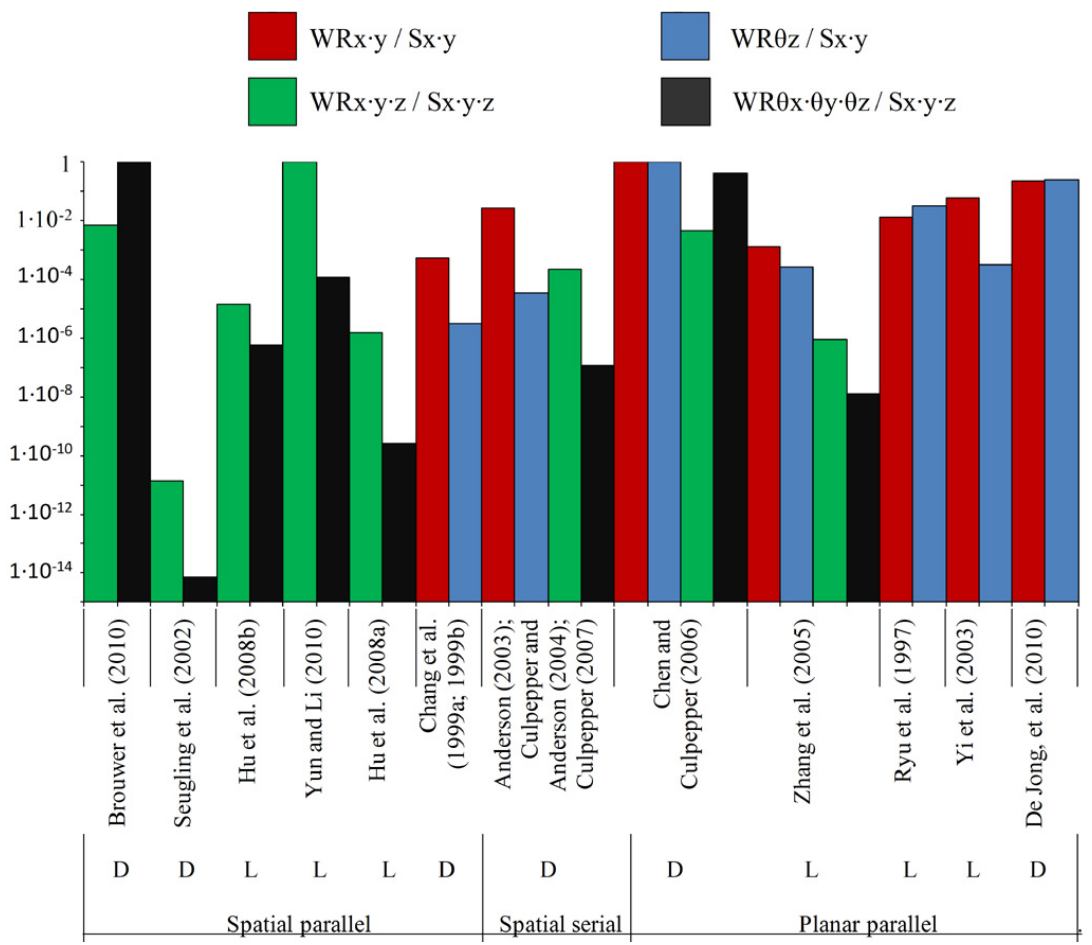

Figure 7. The ratios between translation, or rotation, and size for each compliant stage, if data was available. The ratios were normalized to the largest in the group, shown in logarithmic scale. The mechanisms use distributed compliance (D) or lumped compliance (L).

expected. Also in spatial structures there is no clear relation between working range and flexure type.

In theory, flexures with distributed compliance have a larger range of motion than flexures with lumped compliance. But also lumped compliant flexures were designed such that the complete stage had a large range of motion, using amplifiers in the stage (e.g. the legs in the spatial stages or the 3-revolute-revolute-revolute structure in planar stages act as amplifiers). Most of the stages with lumped compliance are based on these kinds of structures.

In many designs the groups of flexures are arranged $120^{\circ}$ of each other. With a minimum of three equally distributed compliant structures, it is possible to create both translation and rotation of the whole stage, using only translation actuation. In other words, with minimal three $1 \mathrm{DoF}$ compliant structures it is possible to create a $3 \mathrm{DoF}$ stage. Due to this arrangement many stages were highly symmetric. This is to decrease the effect of the temperature gradient on accuracy of the design (Ryu et al., 1997).

From the results it appears that most of the $6 \mathrm{DoF}$ spatial compliant structures are non-monolithic. Some $3 \mathrm{DoF}$ planar structures are promising when implemented in a $6 \mathrm{DoF}$ stage.

All the SBCMs, except one, have distributed compliance and use elastic elements to balance strain energy in the mechanism. The elastic elements (springs and compliant flexures) have been preloaded to store the strain energy, creating zero stiffness. However, pre-stressing of the elastic elements is a challenge and gives difficulties in the design of statically balanced monolithic structures.

For further illustration, the ratios of the statically balanced stroke and compensated force relative to the size of the balancing mechanism is shown in Fig. 8. The compliant micro mechanisms (category 3 of SBCMs) have the largest ratios for statically balanced stroke relative to the size, while this ratio for compensated force relative to the size is still above the average of the other works. The largest ratio for compensated force relative to the size of the balancing mechanism is again for the category 3 of SBCMs. It may be concluded that a balancing mechanism based on buckling plates have great advantages to compensate relative large forces in a relative large stroke, compared to the size. The design with the non-compliant balancing mechanism (category 1 of SBCMs) has the smallest ratio for balanced stroke relative to the size. The preloaded plates shows less efficiency in terms of compensated force and balanced stroke relative to the average, however in all above case, further research is needed as only a few designs were available.

There are few examples of 6 DoF SBMs, but these are all spatial structures, which could be modeled with lumped compliance, balancing gravity forces. No example is available for SBCMs with lumped compliance. Combining SBCMs with lumped compliance, or redesigning an existing $6 \mathrm{DoF}$ SBM, using distributed compliance and balancing strain energy, needs further research and will probably results in a complete new stage design. 

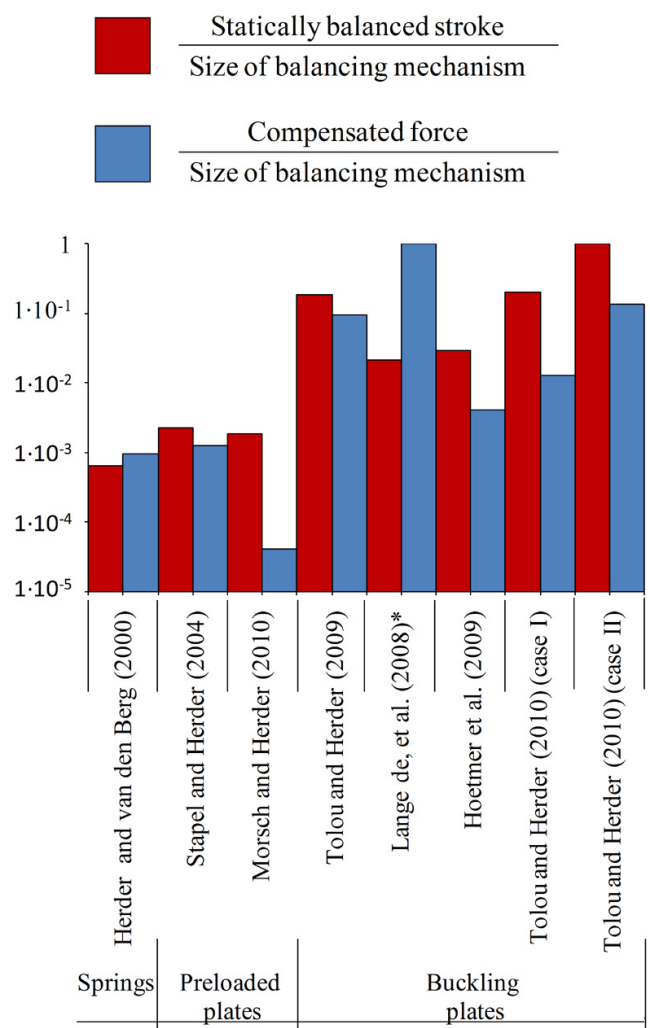

Figure 8. The ratios of statically balanced stroke and compensated force relative to the size of the balancing mechanism. Note that the ratios were normalized to the largest in the group and shown in logarithmic scale. The balancing mechanism used springs, preloaded plates or buckling plates to balance the mechanism.

* This design has an exceptionally high compensated force, but was never fabricated due to calculated high stresses.

\section{Conclusions}

An overview of existing compliant stages, combining translations and rotations $(3-6 \mathrm{DoF})$, classification and discussion, comparing the ratios between translations, rotations and the size, has been made towards the design of 6 DOF statically balanced compliant stage.

It was found that different types of flexures are used in the planar and spatial stages. From the results there is no clear relation between the range of motion and the type of flexure. Where distributed compliance should have a larger range of motion, the lumped compliance stages use different kind of amplifiers to create a large range of motion. Consequently, it can be concluded that effectively each architecture for $6 \mathrm{DoF}$ compliant stages performed equally well.

Different balancing strategies have been studied, as well as the possibilities to combine $6 \mathrm{DoF}$ compliant stages with static balancing.

The compliant balancing mechanisms using buckling plates (either in micro- or mesoscale) shows the better per- formance in terms of force compensation and stroke of static balancing relative to the size of the balancing mechanism.

It is shown that no $6 \mathrm{DoF}$ statically balanced compliant stage is readily available. The existing statically balanced compliant mechanisms have $1 \mathrm{DoF}$, use pre-stressed elastic elements as balancing mechanism, and have distributed compliance, while all existing non-compliant $6 \mathrm{DoF}$ statically balanced stages can be modeled with lumped compliance. Combining static balancing with a $6 \mathrm{DoF}$ compliant stage needs either a new $6 \mathrm{DoF}$ distributed compliant stage, balanced according to the method for balancing distributed compliance, or a new method to balance a lumped compliant 6 DoF stage.

A promising direction for future research would be to find a strategy to combine a $6 \mathrm{DoF}$ monolithic compliant stage with static balancing.

Acknowledgements. This research is part of VIDI Innovational Research Incentives Scheme grant for the project "Statically balanced compliant mechanisms", NOW-STW 7583.

The authors thank Jerry Peijster for his good revisions on this work.

Edited by: C. Kim

Reviewed by: two anonymous referees

\section{References}

Ananthasuresh, G. K. and Kota, S.: Designing compliant mechanisms, Mech. Eng., 117, 93-96, 1995.

Anderson, G. A. B.: A six degree of freedom flexural positioning stage, M.S. thesis, Massachusetts Institute of Technology, Cambridge, USA, 136 pp., 2003.

Brouwer, D. M., de Jong, B. R., and Soemers, H. M. J. R.: Design and modeling of a six DOFs MEMS-based precision manipulator, Precis. Eng., 34, 307-319, 2010.

Chang, S. H., Tseng, C. K., and Chien, H. C.: An ultra-precision $\mathrm{XY} \theta \mathrm{z}$ piezo-micropositioner part I: Design and analysis, IEEE T. Ultrason. Ferr., 46, 897-905, 1999a.

Chang, S. H., Tseng, C. K., and Chien, H. C.: An ultra-precision $\mathrm{XY} \theta \mathrm{z}$ piezo-micropositioner part II: Experiment and performance, IEEE T. Ultrason. Ferr., 46, 906-912, 1999b.

Chao, D., Zong, G., and Liu, R.: Design of a 6-DOF compliant manipulator based on serial-parallel architecture, in: Proceeding of the 2005 IEEE/ASME International Conference on Advanced Intelligent Mechatronics, Monterey, California, USA, 24-28 July 2005, 765-770, 2005.

Chen, S. C. and Culpepper, M. L.: Design of a six-axis micro-scale nanopositioner- $\mu$ HexFlex, Precis. Eng., 30, 314-324, 2006.

Choi, K. B. and Lee, J. J.: Passive compliant wafer stage for singlestep nano-imprint lithography, Rev. Sci. Instrum., 76, 075106, doi:10.1063/1.1948401, 2005

Culpepper, M. L.: Multiple degree of freedom compliant mechanism, patent US2006252297, 2006.

Culpepper, M. L. and Anderson, G.: Design of a low-cost nanomanipulator which utilizes a monolithic, spatial compliant mechanism, Precis. Eng., 28, 469-482, 2004. 
Culpepper, M. L. and Golda, D.: 6-Axis electromagneticallyactuated meso-scale nanopositioner, patent US2007220882, 2007.

Ebert-Uphoff, I. and Johnson, K.: Practical considerations for the static balancing of mechanisms of parallel architecture, P. I. Mech. Eng. K.-J. Mul., 216, 73-85, 2002.

Ebert-Uphoff, I., Gosselin, C. M., and Laliberté, T.: Static balancing of spatial parallel platform mechanisms - revisited, Mech. Des.T. ASME, 122, 43-51, 2000.

Eijk van, J. A. and Dijksman, J. F.: Plate spring mechanism with constant negative stiffness, Mech. Mach. Theory, 14, 1-9, 1979.

Espacenet: http://www.espacenet.com/, last acces: 25 January 2011.

Gallego, J. A. and Herder, J. L.: Synthesis methods in compliant mechanisms: an overview, in: Proceedings of the ASME 2009 International Design Engineering Technical Conferences \& Computers and Information in Engineering Conference, San Diego, California, USA, 30 August-2 September 2009, DETC2009-86845, 2009.

Gao, P. and Swei, S. M.: Six-degree-of-freedom micro-manipulator based on piezoelectric translators, Nanotechnology, 10, 447452, 1999.

Gosselin, C. M. and Wang, J.: Static balancing of spatial sixdegree-of-freedom parallel mechanisms with revolute actuators, J. Robotic Syst., 17, 159-170, 2000.

Helmer, P., Mabillard, Y., Clavel, R., and Bottinelli, S.: High precision apparatus for imposing or measuring a position or a force, patent US20040255696, 2004.

Herder, J. L.: Energy-free systems: theory, conception, and design of statically balanced spring mechanisms, Ph.D. thesis, Delft University of Technology, Delft, The Netherlands, 248 pp., 2001.

Herder, J. L. and van den Berg, F. P. A.: Statically balanced compliant mechanisms (SBCM's), an example and prospects, in: Proceedings of ASME 2000 Design Engineering Technical Conferences and Computers and Information in Engineering Conference, Baltimore, Maryland, USA, 10-13 September 2000, DETC2000/MECH-14144, 2000.

Hoetmer, K., Herder, J. L., and Kim, C. J.: A building block approach for the design of statically balanced compliant mechanisms, in: Proceedings of ASME 2009 International Design Engineering Technical Conferences \& Computers and Information in Engineering Conference, San Diego, California, USA, 30 August-2 September 2009, DETC2009-87451, 2009.

Howell, L. L.: Compliant mechanisms, John Wiley \& Sons, New York USA, 459 pp., 2001.

Hu, K., Kim, J. H., Schmiedeler, J., and Menq, C. H.: Design, implementation, and control of a six-axis compliant stage, Rev. Sci. Instrum., 79, 025105, doi:10.1063/1.2841804, 2008a.

Hu, Y. H., Lin, K. H., Chang, S. C., and Chang, M.: Design of a compliant micromechanism for optical-fiber alignment, Key Eng. Mat., 381-382, 141-144, 2008b.

Jong de, B. R., Brouwer, D. M., de Boer, M. J., Jansen, H. V., Soemers, H. M. J. R., and Krijnen, G. J. M.: Design and fabrication of a planar three-DOFs MEMS-based manipulator, J. Microelectromech. S., 19, 1116-1130, 2010.

Lange de, D. J. B. A., Langelaar, M., and Herder, J. L.: Towards the design of a statically balanced compliant laparoscopic grasper using topology optimization, in: Proceedings of the ASME 2008 International Design Engineering Technical Con- ferences \& Computers and Information in Engineering Conference, Brooklyn, New York, USA, 3-6 August 2008, DETC200849794, 2008

Leblond, M. and Gosselin, C. M.: Static balancing of spatial and planar parallel manipulators with prismatic actuators, in: Proceedings of the 1998 ASME Design Engineering Technical Conferences, Atlanta, Georgia, USA, 13-16 September 1998, DETC98/MECH-5963, 1998.

Lee, C. W. and Kim, S. W.: An ultraprecision stage for alignment of wafers in advanced microlithography, Precis. Eng., 21, 113-122, 1997.

Liang, Q., Zhang, D., Chi, Z., Song, Q., Ge, Y., and Ge, Y.: SixDOF micro-manipulator based on compliant parallel mechanism with integrated force sensor, Robot. CIM-Int. Manuf., 27, 124134, 2011.

Liu, X. J., Wang, J., Gao, F., and Wang, L. P.: On the design of 6-DOF parallel micro-motion manipulators, in: Proceedings of the 2001 IEEE/RSJ International Conference on Intelligent Robots and Systems, Maul, Hawaii, USA, 29 October-3 November 2001, 343-348, 2001.

Lobontiu, N.: Compliant mechanisms: design of flexure hinges, CRC Press LLC, Boca Raton, Florida, USA, 447 pp., 2003.

Lu, T. F., Handley, D. C., Yong, Y. K., and Eales, C.: A three-DOF compliant micromotion stage with flexure hinges, Ind. Robot, 31 , 355-361, 2004.

Moon, Y. M. and Kota, S.: Design of compliant parallel kinematic machines, in: Proceedings of the ASME 2002 Design Engineering Technical Conferences and Computer and Information in Engineering Conference, Montreal, Canada, 29 September-2 October 2002, DETC2002/MECH-34204, 2002.

Morsch, F. M. and Herder, J. L.: Design of a generic zero stiffness compliant joint, in: Proceedings of the ASME 2010 International Design Engineering Technical Conferences \& Computers and Information in Engineering Conference, Montreal, Quebec, Canada, 15-18 August 2010, DETC2010-28351, 2010.

Nieuwenhuis, C.: Thermal behavior Mapper Short Stroke, restricted internal report, Demcon Advanced Mechatronics B.V., Oldenzaal, The Netherlands, 16 pp., 2010.

Park, S. R. and Yang, S. H.: A mathematical approach for analyzing ultra precision positioning system with compliant mechanism, J. Mater. Process. Tech., 164-165, 1584-1589, 2005.

Powell, K. M. and Frecker, M. I.: Method for optimization of a nonlinear static balance mechanism, with application to ophthalmic surgical forceps, in: Proceedings of the ASME 2005 International Design Engineering Technical Conferences \& Computers and Information in Engineering Conference, Long Beach, California, USA, 24-28 September 2005, DETC2005-84759, 2005.

Ryu, J. W., Gweon, D. G., and Moon, K. S.: Optimal design of a flexure hinge based $\mathrm{Xy} \theta$ wafer stage, Precis. Eng., 21, 18-28, 1997.

Scopus: http://www.scopus.com/, last acces: 25 January 2011.

Seugling, R. M., Lebrun, T., Smith, S. T., and Howard, L. P.: A sixdegree-of-freedom precision motion stage, Rev. Sci. Instrum., 73, 2462-2468, 2002.

Shekarforoush, S. M. M., Eghtesad, M., and Farid, M.: Design of statically balanced six-degree-of-freedom parallel mechanisms based on tensegrity system, in: Proceedings of the ASME 2009 International Mechanical Engineering Congress \& Exposition, Lake Buena Vista, Florida, USA, 13-19 November 2009, 
IMECE2009-12625, 2010.

Sjoerdsma, W., Herder, J. L., Horward, M. J., Jansen, A., Bannenberg, J. J. G., and Grimbergen, C. A.: Force transmission of laparoscopic grasping instruments, Minim. Invasiv. Ther., 6, 274 278, 1997.

Stapel, A. and Herder, J. L.: Feasibility study of a fully compliant statically balanced laparoscopic grasper, in: Proceedings of the ASME 2004 Design Engineering Technical Conferences and Computers and Information in Engineering Conference, Salt Lake City, Utah, USA, 28 September-2 October, DETC200457242, 2004.

Stewart, D.: A platform with six degrees of freedom, Proceedings of the Institution of Mechanical Engineers, 180, 371-386, 1965.

Streit, D. A.: Spatial manipulator and six-degree-of-freedom platform spring equilibrator theory, in: Proceedings of the Second National Conference on Applied Mechanisms and Robotics, 1991.

Sun, L., Wang, J., and Wang, Z.: Six-freedom precision paralleled robot, patent CN2576434, 2003.

Sun, S.: Research of nanometer positioning stage with six degree of freedom based on binary actuation principle, in: Proceeding of MircoNanoChina07, Sanya, Hainan, China, 10-13 January 2007, 1639-1647, 2007.

Tian, Y., Shirinzadeh, B., and Zhang, D.: Design and dynamics of a 3-DOF flexure-based parallel mechanism for micro/nano manipulation, Microelectron. Eng., 87, 230-241, 2010.

Tolou, N. and Herder, J. L.: Concept and modeling of a statically balanced compliant laparoscopic grasper, in: Proceedings of ASME 2009 International Design Engineering Technical Conferences \& Computers and Information in Engineering Conference, San Diego, California, USA, 30 August-2 September 2009, DETC2009-86694, 2009.

Tolou, N. and Herder, J. L.: Statically balanced compliant micro mechanisms (SM-MEMS): concepts and simulation, in: Proceedings of the ASME 2010 International Design Engineering Technical Conferences \& Computers and Information in Engineering Conference, Montreal, Quebec, Canada, 15-18 August 2010, DETC2010-28406, 2010.
Trease, B. Dede, E.: Statically-balanced compliant four-bar mechanism for gravity compensation, in: 2004 ASME Student Mechanism Design Competition, 2004.

Wang, H. and Zhang, X.: Input coupling analysis and optimal design of a 3-DOF compliant micro-positioning stage, Mech. Mach. Theory, 43, 400-410, 2008.

Wang, L., Rong, W., Sun, L., and Jiao, J.: Analysis and design of a three-limb six degree-of-freedom parallel micromanipulator with flexure hinges, in: Proceeding of MircoNanoChina07, Sanya, Hainan, China, 10-13 January 2007, 1561-1566, 2007.

Wang, S. C., Hikita, H., Kubo, H., Zhao, Y. S., Huang, Z., and Ifukube, T.: Kinematics and dynamics of a 6 degree-of-freedom fully parallel manipulator with elastic joints, Mech. Mach. Theory, 38, 439-461, 2003.

Wang, Y., Liu, Z., Bo, F., and Zhu, J.: Design and research of 5-DOF Integrated nanopositioning stage, Zhongguo Jixie Gongcheng/China Mechanical Engineering, 16, 1317-1321, 2005.

Willson, C. G. and Roman, B. J.: The future of lithography: Sematech litho forum 2008, ACS Nano, 2, 1323-1328, 2008.

Xiaohui, J., Yanling, T., and Dawei, Z.: Six-freedom-degree precision positioning table for nano-imprint lithography system, patent CN101726997, 2010.

Xuchu, J. and Qianfeng, Q.: Precise positioning platform with six freedom of motion, patent CN101488371, 2009.

Yi, B. J., Chung, G. B., Na, H. Y., Kim, W. K., and Suh, I. H.: Design and experiment of a 3-DOF parallel micromechanism utilizing flexure hinges, IEEE T. Robotic. Autom., 19, 604-612, 2003.

Yun, Y. and Li, Y.: Design and analysis of a novel 6-DOF redundant actuated parallel robot with compliant hinges for high precision positioning, Nonlinear Dynam., 61, 829-845, 2010.

Zhang, D. Y., Ono, T., and Esashi, M.: Piezoactuator-integrated monolithic microstage with six degrees of freedom, Sensor. Actuat. A-Phys., 122, 301-306, 2005. 